\title{
Photonic metamaterials by direct laser writing and silver chemical vapour deposition
}

\author{
MICHAEL S. RILL ${ }^{1 \star}$, CHRISTINE PLET ${ }^{1}$, MICHAEL THIEL ${ }^{1}$, ISABELLE STAUDE ${ }^{1}$, \\ GEORG VON FREYMANN ${ }^{1}, 2$, STEFAN LINDEN ${ }^{1,2}$ AND MARTIN WEGENER ${ }^{1,2}$ \\ ${ }^{1}$ Institut für Angewandte Physik, Universität Karlsruhe (TH), Wolfgang-Gaede-Straße 1, D-76131 Karlsruhe, Germany \\ ${ }^{2}$ Institut für Nanotechnologie, Forschungszentrum Karlsruhe in der Helmholtz-Gemeinschaft, D-76021 Karlsruhe, Germany \\ *e-mail: Michael.Rill@physik.uni-karlsruhe.de
}

Metamaterials are artificial materials that-unlike natural substances-enable magnetism to be achieved at optical frequencies $^{1-3}$. The vast majority of photonic metamaterials ${ }^{4,5}$ has been fabricated by electron-beam lithography and evaporation of metal films, both of which are well-established twodimensional (2D) technologies. Although stacking of three ${ }^{6}$ or four ${ }^{7}$ functional layers made using these methods has been reported, a truly 3D fabrication approach would be preferable for 3D photonic metamaterials. Here, we report first steps in this direction by using a combination of direct laser writing ${ }^{8,9}$ and silver chemical vapour deposition ${ }^{10,11}$ - the $3 \mathrm{D}$ analogues of electron-beam lithography and evaporation, respectively. The optical characterization of a planar test structure composed of elongated split-ring resonators is in good agreement with theory. Retrieval of the effective optical parameters reveals the importance of bi-anisotropy. Once suitable theoretical blueprints are available, our fabrication approach will enable rapid prototyping of truly $3 \mathrm{D}$ photonic metamaterials.

The emerging field of photonic metamaterials is driven by the fascinating visions of perfect lenses ${ }^{12}$, optical cloaking ${ }^{13,14}$ or quantum levitation ${ }^{15}$. Realizing these visions clearly ultimately requires large-scale three-dimensional (3D) metamaterials operating at optical frequencies. One of the major challenges concerns the (realistic) design and the nanofabrication of such structures. The latter is related to the fabrication of $3 \mathrm{D}$ (dielectric) photonic crystals, for which extensive literature is available (see, for example, the references in a recent review $\left.{ }^{16}\right)$. It has become clear that polymeric templates made using holography and/or direct laser writing (DLW) can subsequently be converted into, for example, silicon structures using chemical vapour deposition (CVD) and/or atomic-layer deposition (ALD). In the context of metamaterials, however, metals are crucial; silver leads to the lowest losses at optical frequencies ${ }^{17}$. Yet, it has been unclear whether structures made using DLW and silver CVD would ever have sufficient quality and whether achievable feature sizes would result in metamaterials operating at optical frequencies. Fortunately, we report here that the answer is positive in both regards. Combined with suitable theoretical blueprints, which still need to be developed, this result means that one of the major hurdles in the field of photonic metamaterials is starting to disappear.
Lithography of 3D polymeric templates by DLW with lateral feature sizes in the $100 \mathrm{~nm}$ range has become routine $\mathrm{e}^{8,9,18}$ and is even available commercially ${ }^{19}$. Our vision is to take such a template and coat it with a silver film of a few tens of nanometres in thickness, for example leading to the 3D arrangement of magnetic split-ring resonators (SRR) shown in Fig. 1a. Several questions arise immediately. (1) Silver films made using these methods are obviously always inherently connected-in sharp contrast to those made by electron-beam lithography. Can meaningful properties, especially a magnetic response, still be achieved? (2) The quality of silver films made using CVD is usually nowhere close to that of films made using high-vacuum evaporation. ALD of silver has never been reported. Can a high-quality metamaterial optical response still be accomplished? To address these questions, we consider the much simpler arrangement of SRRs shown in Fig. 1b. It can be viewed as a 1D lattice of elongated SRRs or simply as a corrugated surface ${ }^{20-22}$.

The fabrication starts with a glass substrate covered with a $2-\mu \mathrm{m}$-thick fully polymerized resist film (SU-8). Another SU-8 film is spun-on, exposed using DLW, post-baked and developed. The resulting SU-8 template is coated with a thin layer of $\mathrm{SiO}_{2}$ (typically a few tens of nanometres) using ALD with $\mathrm{SiCl}_{4}$ as the precursor ${ }^{23}$. The $\mathrm{SiO}_{2}$ surface is subsequently exposed to $\mathrm{O}_{2}$ plasma for $15 \mathrm{~min}$ to activate the surface. The $\mathrm{SiO}_{2}$ layer provides mechanical stability as well as chemical protection for the SU-8 backbone in the following silver CVD process in which the template needs to be heated to $160^{\circ} \mathrm{C}$. Here, the metal-organic precursor $(\mathrm{COD})(\mathrm{hfac}) \mathrm{Ag}(\mathrm{I})$ is sublimed at a temperature of $60^{\circ} \mathrm{C}$; the wall temperature of the CVD chamber is $80^{\circ} \mathrm{C}$. In each static cycle of $40 \mathrm{~min}$ in duration, we deposit about $5 \mathrm{~nm}$ of silver. The samples discussed below have resulted from 10 CVD cycles, equivalent to an estimated silver thickness of about $50 \mathrm{~nm}$. Figure 2 shows electron micrographs of structures made using these methods. Importantly, the structure shown in Fig. $2 \mathrm{~b}$ demonstrates that the coating is uniform around the structure even in $3 \mathrm{D}$-in sharp contrast to a usual 2D evaporation process. The films are somewhat granular but they are connected. This crucial aspect is demonstrated by the fact that the silver films exhibit good d.c. conductivity, by a reflectance $R>95 \%$ in the $1-4 \mu \mathrm{m}$ wavelength range, and by the optical properties to be discussed next.

To determine the optical properties of the metamaterial layer, we have measured normal-incidence transmittance spectra using a 


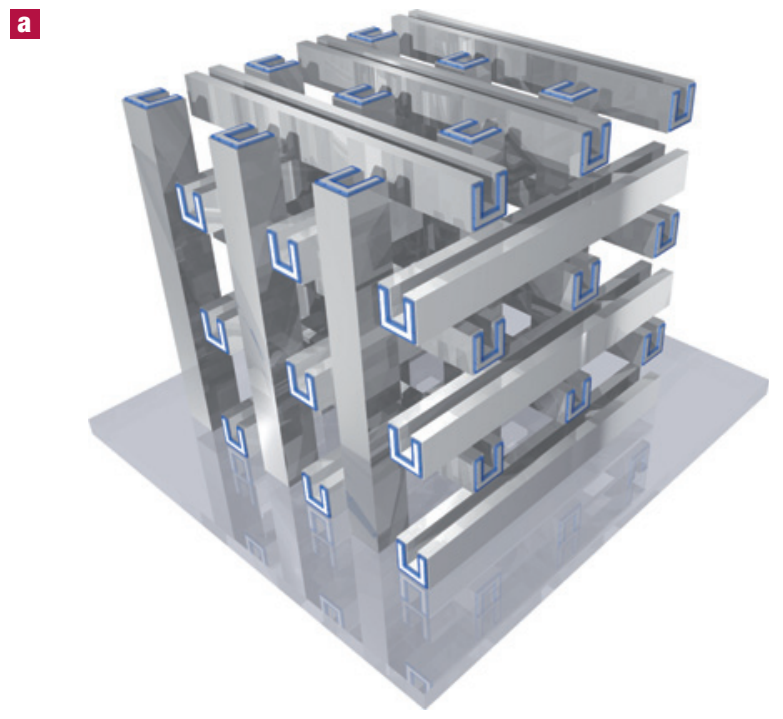

b

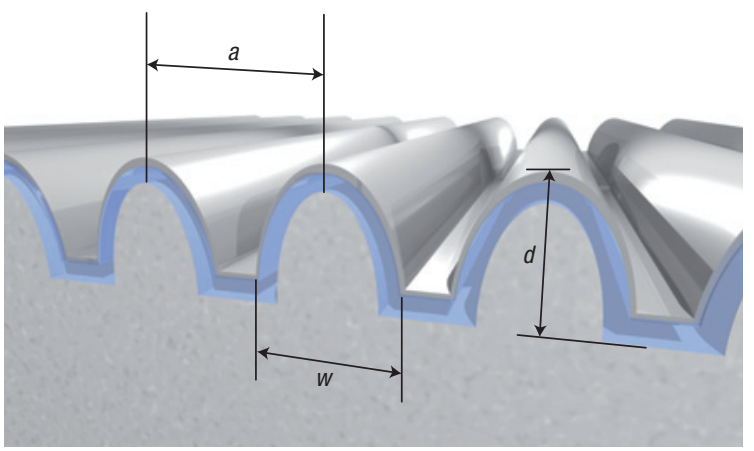

Figure 1 Metamaterial structures. a, A hypothetical 3D arrangement of elongated SRRs. Owing to the anticipated DLW and silver CVD fabrication process, all metal parts are connected. $\mathbf{b}$, A planar lattice of elongated SRRs, again all connected. The latter structure shall serve as a reasonably simple test bed for the novel fabrication approach outlined here. Polymer (light grey), silica (blue) and silver (dark grey, reflective).

Fourier-transform microscope spectrometer (Bruker Equinox 55, near-infrared halogen source and Bruker Hyperion 2000, $\times 36$ Cassegrain objectives, numerical aperture: 0.5 , liquid- $\mathrm{N}_{2}$-cooled InSb detector). Results for the incident light being polarized vertically to the grooves of the elongated SRRs are shown in Fig. 3a. Different heights $d$ correspond to different fundamental resonance frequencies. Intuitively, reducing the height leads to a reduction of the SRR cross-section and thus of its inductance $L$ ( $C$ is its capacitance), and hence to an increase of the SRR $L C$ eigenfrequency (decrease of the wavelength $\lambda$ ) at fixed lattice constant $a=800 \mathrm{~nm}$. Notably, the structure with $d=740 \mathrm{~nm}$ (blue curve) reveals a ratio of about $\lambda / a=4$, which is well separated from the Rayleigh anomalies and the Bragg condition, that is, the effective-medium approximation is well justified.

To enable a direct comparison with the theoretical ideal, we have carried out 3D finite-difference time-domain calculations using the software package CST Microwave Studio. The refractive indices of dielectrics used are $n_{\mathrm{SU}-8}=1.55$ and $n_{\mathrm{SiO}_{2}}=1.50$. Here, the silver film thickness, $t_{\mathrm{Ag}}$, has been used as an adjustable parameter, leading to $t_{\mathrm{Ag}}=30 \mathrm{~nm}$. As usual, the silver optical properties are described by the free-electron Drude model with literature parameters ${ }^{6}$, that is, plasma frequency

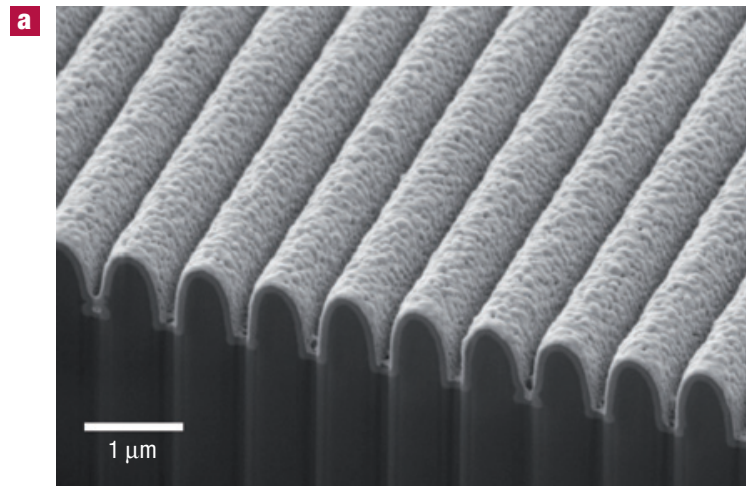

b

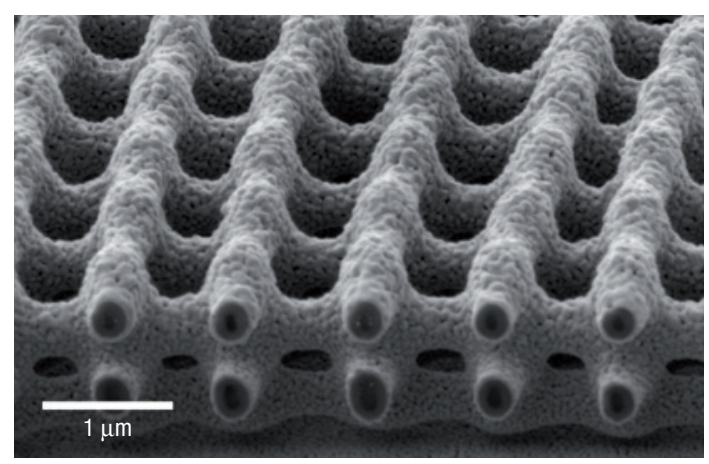

Figure 2 Electron micrographs of fabricated structures. The oblique views show structures that have been cut by a focused-ion beam after fabrication to reveal the interior (rather than an edge). a, Metamaterial corresponding to the design schematically shown in Fig. $1 \mathrm{~b}$. The focused-ion-beam cut reveals the $\mathrm{SiO}_{2}$ layer between the SU-8 template and the silver coating. b, A 3D structure composed of bars. Note that the silver coating covers the bars all around.

$\omega_{\mathrm{pl}}=1.37 \times 10^{16} \mathrm{~s}^{-1}$ and collision frequency $\omega_{\mathrm{col}}=8.50 \times 10^{13} \mathrm{~s}^{-1}$. Figure $3 \mathrm{~b}$ shows numerically calculated transmittance spectra corresponding to the experiment and plotted on the same horizontal and vertical scales. The overall agreement is good, especially regarding the position of the magnetic resonance, the depth of this resonance and the general qualitative trends with SRR height $d$. Quantitative deviations can partly be due to systematic variations in the thickness of the silver film. Furthermore, the statistical roughness of the metal film can lead to Rayleigh scattering. This effect tends to decrease the transmittance on the short-wavelength side of the spectrum, especially for effectively thicker structures (larger $d$ ). Finally, it should be noted that the Cassegrain optics in the experiment collects opening angles between $15^{\circ}$ and $30^{\circ}$ around the surface normal, whereas the calculations refer to strictly normal incidence. Our finite-difference time-domain approach does not allow calculations for oblique incidence. However, we have also carried out extra calculations based on a scattering-matrix approach, for oblique incidence of light, but for a simplified rectangular SRR shape. Here, we find changes in the transmittance spectra for angles between $0^{\circ}$ and $30^{\circ}$ that are comparable to the mentioned deviations between experiment and theory in Fig. 3a and b, respectively.

Altogether, we have sufficient confidence in the theory to extract the effective optical parameters on the basis of these calculations. Extreme caution has to be taken at this point. The metamaterial structure (Fig. 1b or Fig. 2a) clearly lacks inversion symmetry along the propagation direction. Thus, a parameter retrieval ${ }^{24}$ in terms of complex permittivity $\varepsilon$ and complex permeability $\mu$ 


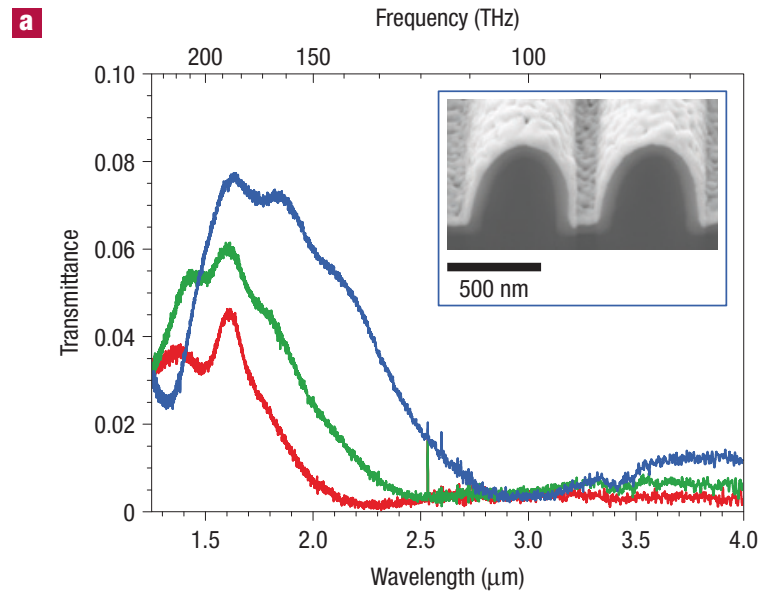

b

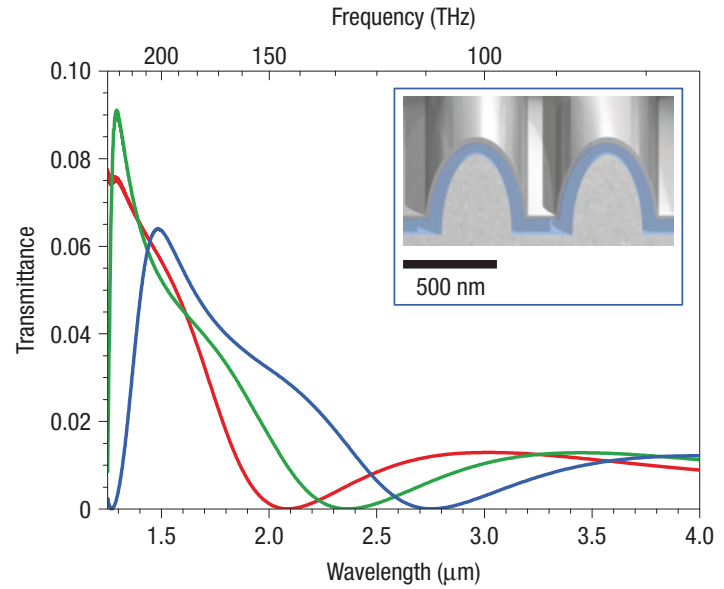

Figure 3 Normal-incidence optical transmittance spectra. a, Experiment. b, Corresponding theory. The incident linear polarization is perpendicular to the grooves shown in Figs $1 \mathrm{~b}$ and $2 \mathrm{a}$. The insets show an electron micrograph of a corresponding structure (blue curve) and an illustration of the geometry used in the calculations, respectively. Mainly, the height $d$ of the structure (Fig. 1b) has been varied: $d=580 \mathrm{~nm}$ (red curve), $d=640 \mathrm{~nm}$ (green curve) and $d=740 \mathrm{~nm}$ (blue curve). Owing to the fabrication process, the width $w$ also changes: $w=590 \mathrm{~nm}$, $w=570 \mathrm{~nm}$ and $w=500 \mathrm{~nm}$, respectively.

alone is generally not possible as this always leads to a symmetric reflectance, whereas the actual reflectance is not symmetric, that is, it depends on which side it is taken from. The general underlying physics of bi-anisotropy has been discussed in the literature $e^{25,26}$ (alternatively, the behaviour can be expressed in terms of a nonlocal response $\mathrm{s}^{27}$ ). Here, we are not interested in the general tensor formulation $^{25}$ and can restrict ourselves to the $1 \mathrm{D}$ situation. If the incident light is polarized vertically to the grooves of the elongated SRRs, the relation between the relevant vector components of the electromagnetic fields in the Maxwell equations can be simplified to

$$
\left(\begin{array}{l}
D \\
B
\end{array}\right)=\left(\begin{array}{cc}
\varepsilon_{0} \varepsilon & -i c_{0}^{-1} \xi \\
+i c_{0}^{-1} \xi & \mu_{0} \mu
\end{array}\right)\left(\begin{array}{c}
E \\
H
\end{array}\right) \text {. }
$$

The complex refractive index $n$ results from $n^{2}=\varepsilon \mu-\xi^{2} . \varepsilon_{0}$ is the vacuum permittivity, $\mu_{0}$ is the vacuum permeability and $c_{0}$ is the vacuum speed of light. As usual, the permittivity $\varepsilon$ describes excitation of electric dipoles by the electric component of the incident electromagnetic field, and $\mu$ describes the excitation of magnetic dipoles by the magnetic component. The bi-anisotropy

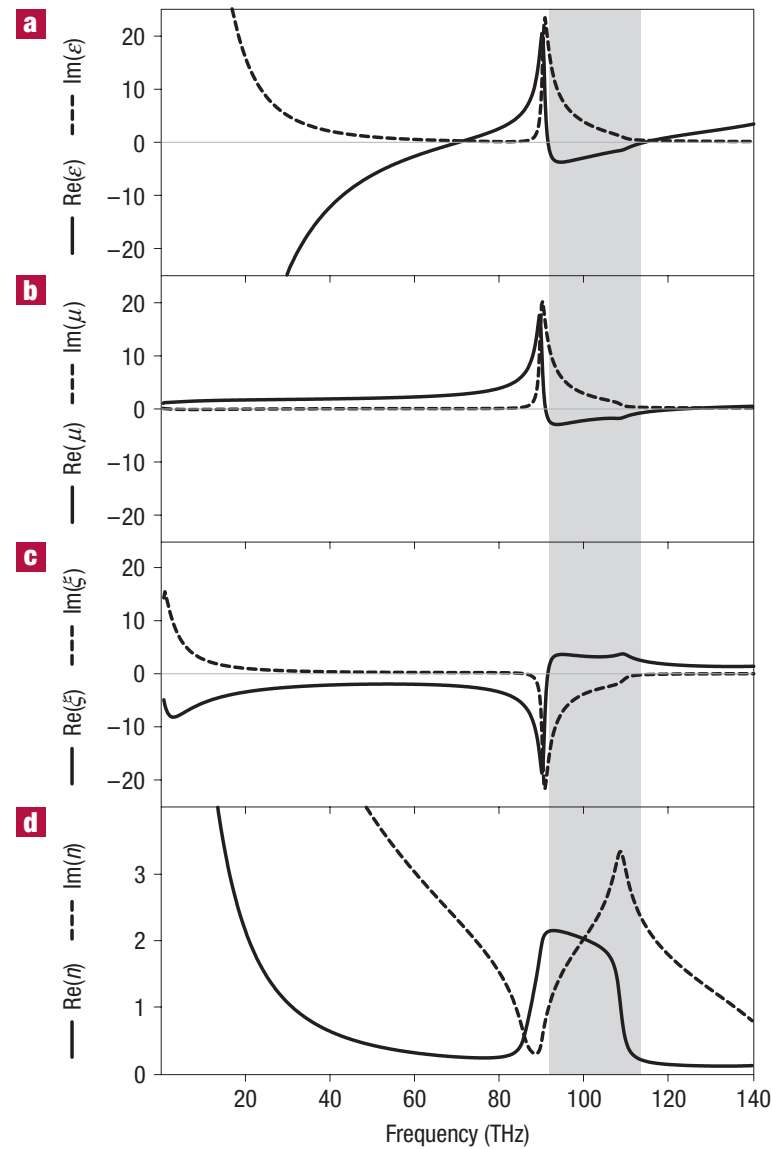

Figure 4 Retrieved effective metamaterial parameters. Real and imaginary parts of the complex parameters. a, Electric permittivity $\varepsilon$. b, Magnetic permeability $\mu$. $\mathbf{c}$, Bi-anisotropy parameter $\xi$. d, Refractive index $n$. Note the magnetic resonance at a frequency of around $100 \mathrm{THz}$ ( $3 \mu \mathrm{m}$ wavelength) and that $\operatorname{Re}(n)>0$, whereas $\operatorname{Re}(\varepsilon)<0$ and $\operatorname{Re}(\mu)<0$ owing to the substantial influence of $\xi$. Parameters correspond to Fig. 3b, $d=740 \mathrm{~nm}$ (blue curve).

parameter $\xi$ (see off-diagonal terms in the matrix) describes the excitation of magnetic dipoles by the electric component of the field and vice versa. Reciprocity demands that the two off-diagonal terms are identical up to the minus sign.

Resulting retrievals (see the Methods section) are shown in Fig. 4 . The magnetic permeability $\mu$ does exhibit the anticipated magnetic resonance with $\operatorname{Re}(\mu)<0$ in a certain frequency interval, showing that our primary goal of fabricating a magnetic metamaterial using DLW and silver CVD has been accomplished. At first sight, ignoring the effects of bi-anisotropy, it might be tempting to conclude that the real part of the refractive index should be negative from the fact that the condition $\operatorname{Re}(\varepsilon)<0$ holds in an overlapping frequency interval. Yet, the influence of bi-anisotropy is far from being a detail or a correction and rather gives a positive resonance in $\operatorname{Re}(n)>0$.

Metamaterial losses can be quantified by the ratio of the real and imaginary parts of the refractive index, $\operatorname{Re}(n) / \operatorname{Im}(n)$ (Fig. $4 \mathrm{~d}$ ). This ratio reaches a maximum of about five. Thus, the very low transmittance of the structure shown in Fig. 3a,b is not a result of losses, but rather a result of the large impedance mismatch between the metamaterial and vacuum or glass substrate, respectively.

In summary, we have shown that high-quality magnetic metamaterials at near-infrared frequencies can be fabricated using 
the combination of direct laser writing of polymer templates and silver chemical vapour deposition-the $3 \mathrm{D}$ analogues of $2 \mathrm{D}$ electron-beam lithography and 2D evaporation. Our approach enables rapid prototyping of complex 3D photonic metamaterials. Currently, however, we are facing a bottleneck in that theory has not yet provided blueprints for 3D metamaterials compatible with this approach. We hope that such theoretical progress will be stimulated by our work, as it is becoming increasingly clear that truly $3 \mathrm{D}$ photonic metamaterials of the future will very likely not just be miniaturized versions of their microwave counterparts ${ }^{28}$.

\section{METHODS}

The Fresnel equations of a bi-anisotropic material for normal incidence enable calculation of the complex transmittance coefficients $t_{\text {air }}$ and $t_{\text {sub }}$ (incident light propagates at first through the substrate) as well as the two complex reflectance coefficients $r_{\text {air }}$ and $r_{\text {sub }}$ (light is reflected at the substrate-structure interface). Inversion of these equations leads to the relative impedances $z_{ \pm}=\left(-b \mp \sqrt{b^{2}-4 a c}\right) /(2 a)$, with

$$
\begin{gathered}
a=t_{\mathrm{air}} t_{\mathrm{sub}}-\left(1-r_{\mathrm{air}}\right)\left(1-r_{\mathrm{sub}}\right), \\
b=\left(z_{\mathrm{air}}-z_{\mathrm{sub}}\right)\left(t_{\mathrm{air}} t_{\mathrm{sub}}+1-r_{\mathrm{air}} r_{\mathrm{sub}}\right)+\left(z_{\mathrm{air}}+z_{\mathrm{sub}}\right)\left(r_{\mathrm{air}}-r_{\mathrm{sub}}\right), \\
c=z_{\mathrm{air}} z_{\mathrm{sub}}\left(-t_{\mathrm{air}} t_{\mathrm{sub}}+\left(1+r_{\mathrm{air}}\right)\left(1+r_{\mathrm{sub}}\right)\right),
\end{gathered}
$$

and the refractive index $n$ from

$$
\begin{aligned}
\cos \left(n k_{0} d\right)= & \frac{t_{\text {air }}}{2}\left[\frac{\left(1-\frac{z_{-}}{z_{\text {sub }}}\right)}{\left(1-\frac{z_{-}}{z_{\text {air }}}\right)+r_{\text {air }}\left(1+\frac{z_{-}}{z_{\text {air }}}\right)}\right. \\
& \left.+\frac{\left(1-\frac{z_{+}}{z_{\text {sub }}}\right)}{\left(1-\frac{z_{+}}{z_{\text {air }}}\right)+r_{\text {air }}\left(1+\frac{z_{+}}{z_{\text {air }}}\right)}\right] .
\end{aligned}
$$

$k_{0}$ is the vacuum wavenumber, $d$ is the thickness of the effective medium, $z_{\text {sub }}=Z_{\text {sub }} / Z_{0}$ is the relative substrate impedance and $z_{\text {air }}=Z_{\text {air }} / Z_{0}=1$ is the relative vacuum impedance with the absolute vacuum impedance $Z_{0}=\sqrt{\mu_{0} / \varepsilon_{0}}$. Clearly, the inverse of equation (1) has different roots owing to the inverse cosine. Regarding selecting the correct root, we proceed as in the symmetric case $\mathrm{e}^{24}$. Finally, for the material parameters, we derive $\xi=i n\left(z_{-}+z_{+}\right) /\left(z_{-}-z_{+}\right), \mu=z_{+}(n-i \xi)$ and $\varepsilon=(n+i \xi) / z_{+}$.

\section{Received 10 March 2008; accepted 16 April 2008; published 11 May 2008.}

\section{References}

1. Pendry, J. B., Holden, A. J. Robbins, D. J. \& Stewart, W. J. Magnetism from conductors and enhanced nonlinear phenomena. IEEE Trans. Microw. Theory Tech. 47, 2075-2084 (1999).

2. Linden, S. et al. Magnetic response of metamaterials at 100 Terahertz. Science 306, 1351-1353 (2004).
3. Cai, W. et al. Metamagnetics with rainbow colors. Opt. Express 15, 3333-3341 (2007).

4. Shalaev, V. M. Optical negative-index metamaterials. Nature Photon. 1, 41-48 (2007).

5. Soukoulis, C. M., Linden, S. \& Wegener, M. Negative refractive index at optical wavelengths. Science 315, 47-49 (2007).

6. Dolling, G., Wegener, M. \& Linden, S. Realization of a three-functional-layer negative-index photonic metamaterial. Opt. Lett. 32, 551-553 (2007).

7. Liu, N. et al. Three-dimensional photonic metamaterials at optical frequencies. Nature Mater. 7 , 31-37 (2008).

8. Kawata, S., Sun, H.-B., Tanaka, T. \& Takada, K. Finer features for functional microdevices. Nature 412, 697-698 (2001).

9. Deubel, M. et al. Direct laser writing of three-dimensional photonic-crystal templates for telecommunications. Nature Mater. 3, 444-447 (2004).

10. Hampden-Smith, M. J. \& Kodas, T. T. Chemical vapor deposition of metals: Part 1. An overview of CVD processes. Chem. Vap. Deposition 1, 8-23 (1995).

11. Eisenbraun, E. T., Klaver, A., Patel, Z., Nuesca, G. \& Kaloyeros, A. E. Low temperature metalorganic chemical vapor deposition of conformal silver coatings for applications in high aspect ratio structures. J. Vac. Sci. Technol. B 19, 585-588 (2001).

12. Pendry, J. B. Negative refraction makes a perfect lens. Phys. Rev. Lett. 85, 3966-3969 (2000).

13. Pendry, J. B., Schurig, D. \& Smith, D. R. Controlling electromagnetic fields. Science 312, 1780-1782 (2006).

14. Schurig, D. et al. Metamaterial electromagnetic cloak at microwave frequencies. Science 314, 977-980 (2006)

15. Leonhardt, U. \& Philbin, T. G. Quantum levitation by left-handed metamaterials. New J. Phys. 9 , 254:1-11 (2007)

16. Busch, K. et al. Periodic nanostructures for photonics. Phys. Rep. 444, 101-202 (2007).

17. Johnson, P. B. \& Christy, R. W. Optical constants of the noble metals. Phys. Rev. B 6, 4370-4379 (1972).

18. Formanek, F. et al. Three-dimensional fabrication of metallic nanostructures over large areas by two-photon polymerization. Opt. Express 14, 800-809 (2006).

19. <http://www.nanoscribe.de>.

20. Schröter, U. \& Heitmann, D. Grating couplers for surface plasmons excited on thin metal films in the Kretschmann-Raether configuration. Phys. Rev. B 60, 4992-4999 (1999).

21. Zhang, S. et al. Midinfrared resonant magnetic nanostructures exhibiting a negative permeability. Phys. Rev. Lett. 94, 037402 (2005).

22. Schweizer, H. et al. Negative permeability around $630 \mathrm{~nm}$ in nanofabricated vertical meander metamaterials. Phys. Status Solidi A 204, 3886-3900 (2007).

23. Ehrlich, D. J. \& Melngailis, J. Fast room-temperature growth of $\mathrm{SiO}_{2}$ films by molecular-layer dosing. Appl. Phys. Lett. 58, 2675-2677 (1991).

24. Smith, D. R., Schultz, S., Markoš, P. \& Soukoulis, C. M. Determination of effective permittivity and permeability of metamaterials from reflection and transmission coefficients. Phys. Rev. B 65 195104 (2002).

25. Chen, X., Wu, B.-I., Kong, J. A. \& Grzegorczyk, T. M. Retrieval of the effective constitutive parameters of bianisotropic metamaterials. Phys. Rev. E 71, 046610 (2005).

26. Marqués, R., Medina, F. \& Rafii-El-Idrissi, R. Role of bianisotropy in negative permeability and left-handed metamaterials. Phys. Rev. B 65, 144440 (2002).

27. Bungay, A. R., Svirko, Yu. P. \& Zheludev, N. I. Equivalency of the Casimir and the Landau-Lifshitz approaches to continuous-media electrodynamics and optical activity on reflection. Phys. Rev. B 47, 11730-11735 (1993).

28. Smith, D. R., Pendry, J. B. \& Wiltshire, M. C. K. Metamaterials and negative refractive index. Science 305, 788-792 (2004).

\section{Acknowledgements}

We thank C. M. Soukoulis for stimulating discussions. We acknowledge financial support provided by the Deutsche Forschungsgemeinschaft (DFG) and the State of Baden-Württemberg through the DFGCenter for Functional Nanostructures (CFN) within subprojects A1.4 and A1.5. The project PHOME acknowledges the financial support of the Future and Emerging Technologies (FET) programme within the Seventh Framework Programme for Research of the European Commission, under FET-Open grant number 213390. Also we acknowledge funding by the Bundesministerium für Bildung und Forschung (BMBF). The research of G.v.F. is further supported through a DFG Emmy-Noether fellowship (DFG-Fr 1671/4-3), that of S.L. through a 'Helmholtz-Hochschul-Nachwuchsgruppe' (VH-NG-232).

\section{Author information}

The authors declare competing financial interests: details accompany the full-text HTML version of the paper at www.nature.com/naturematerials. Reprints and permission information is available online at http://npg.nature.com/reprintsandpermissions. Correspondence and requests for materials should be addressed to M.S.R. 